\title{
Interactions of Melatonin and MicroRNAs
}

\author{
Rüdiger Hardeland* \\ Johann Friedrich Blumenbach Institute of Zoology and Anthropology, University of Göttingen, Göttingen, Germany
}

${ }^{*}$ Corresponding author: Rüdiger Hardeland, Johann Friedrich Blumenbach Institute of Zoology and Anthropology, University of Göttingen, Bürgerstr. 50, D-37073 Göttingen, Germany, Tel: +49-551-395414; E-mail: rhardel@gwdg.de

Received Date: February 09, 2018; Accepted Date: February 15, 2018; Published Date: February 19, 2018

Citation: Hardeland R (2018) Interactions of Melatonin and MicroRNAs. Biochem Mol Biol J Vol. 4: No. 1:7.

\section{Abstract}

MicroRNAs (miRNAs) are important players in the modulation of cellular functions and contribute substantially to epigenetic changes in the expression of genes. Moreover, the distribution of miRNAs via exosomes and ectosomes opens a vast field of intraorganismal communication, with the potential of spreading pathological deviations, but also curative effects induced by protective agents. Interactions between melatonin and microRNAs are of particular interest, as melatonin is a highly pleiotropic regulator of numerous functions in every organ. The effects of melatonin in correcting pathological alterations in miRNA composition are reviewed, along with the corresponding reversal of cell biological or physiological functions to normal. Additionally, knowledge on the influence of miRNAs on melatonin formation and expression of a melatonin receptor has been considered. The fields in which melatonin has been shown to influence miRNAs are as diverse as metabolic syndrome, liver steatosis, immunology, amyloid toxicity, progenitor cells, and cancer. Readers are encouraged to contribute to systematic studies on melatonin effects on miRNAs using modern RNA sequencing techniques.

Keywords: Cancer; Inflammation; Long noncoding RNA; Melatonin; MicroRNA; Neurodegeneration

\section{Introduction}

Epigenetic regulation receives increasing attention in cell biology and medicine. In melatonin research, this is an emerging field [1-3]. Among the multiple epigenetic changes, the contribution of microRNAs (miRNAs) as regulators of countless functions is of particular interest [4-9]. The number of mammalian miRNAs is in the range about one thousand, but their modulatory actions extend to the expression of, at least, half of the coding genes, because single miRNAs can sometimes interact with hundreds of mRNAs or their precursors, hnRNAs, or even with the respective antisense transcripts (asRNAs, which belong to the group of long noncoding RNAs, IncRNAs) [4-8]. Target mRNAs, asRNAs or hnRNAs are typically derived from genes that are dynamically regulated or others that are particularly important for the functional state of a cell. The typical and most frequently observed action concerns the silencing by Argonaute (AGO)mediated duplex formation with the target, assembly of a silencing complex (RISC complex) followed by duplex cleavage. However, if an asRNA is the target, this may instead result in the elimination of a blockade. In addition to interactions with other RNAs, some miRNAs have been shown to also target specific toll-like receptors (TLRs) [9-11]. However, this is only possible with TLRs that are capable of binding single-stranded RNAs (ssRNAs) but not double-stranded RNAs (dsRNAs). Binding to TLR7 was first shown for the miRNAs Let-7c and $\operatorname{miR}-21[10]$.

\section{Discussion}

MicroRNAs are also of utmost interest with regard to their modes of distribution from one cell to others. They may enter neighboring cells via gap junctions, but they can be also transmitted by exosomes and ectosomes. These are found in all body fluids and interact specifically with target cells $[9,12]$. Thereby, they are capable of transporting messages over a long distance in the body. As a consequence, the regulatory network in a body is considerably larger and more complex than previously believed.

The actions of miRNAs are not only of physiological relevance, but have an additional pathological dimension and contribute to dysregulation. Numerous diseases including cancer and even mood disorders display changes in composition and amounts of microRNAs. Transmission of dysregulated miRNAs by exosomes or ectosomes to recipient cells is actually discussed as part of disease spreading within the body [9]. In exosomes and ectosomes, miRNAs are typically enriched. One of the reasons is that these vesicles also contain larger RNAs, especially IncRNAs and circular RNAs (circRNAs) that act as miRNA "sponges" by binding a high amount of miRNA molecules [9,13,14].

Regulatory interactions of melatonin with miRNAs are of particular interest as the former acts as a highly pleiotropic regulator molecule that orchestrates countless body functions, both directly and indirectly via central and peripheral circadian oscillators $[15,16]$. Circadian oscillators and even melatonin synthesis are also subject to modulation by miRNAs [2,17-19]. Moreover, melatonin does not only act as a pineal hormone, but is synthesized in many extrapineal organs and in immune 
cells $[15,20]$. The total amount of extrapineally formed melatonin exceeds those in the pineal gland and in the circulation by more than two orders of magnitude $[15,21]$.

Melatonergic signaling can be affected by miRNAs, but the available information is still limited. The melatonin receptor $\mathrm{MT}_{1}$ was downregulated in the context of type 2 diabetes by miR-29b, an effect that counteracted melatonin's beneficial role concerning obesity and metabolic syndrome [22]. Much more data are available for protective effects of melatonin that involve changes in miRNA expression. In a murine model of alcoholic liver disease, protection by melatonin enhanced miR-497 expression, which caused downregulation of the targets Btg2 (B-cell translocation gene 2) and $Y y 1$ (Yin yang 1) [23]. Liver steatosis was reported to be ameliorated by melatonin via miR-23a [24]. In murine model of liver fibrosis, melatonin reduced primary sclerosing cholangitis by downregulating miR-200b in cholangiocytes and stellate cells [25]. Studies on miRNAs in Alzheimer's disease (AD) are of particular interest, because neuroinflammation, which is fueled by $A \beta$ peptides and oligomers, can be partially reversed by melatonin [26]. In a rat scopolamine toxicity model of ADlike memory losses, melatonin reversed an increase of miR-124 and thereby corrected the level of the targeted Egr1 (early growth response protein 1) mRNA [27]. In another AD model, $A \beta_{25-35}$ peptide added to primary cortical neurons caused downregulation miR-132, an effect that was also reversed by melatonin. The normalization of miR-132 is insofar of importance, as this miRNA transmits anti-apoptotic and other protective properties known from melatonin [28]. In neonatal brain inflammation induced in rats by bacterial lipopolysaccharide (LPS), this pro-inflammatory agent upregulated miR-34a, but downregulated miR-146a, and miR-126. Again, these changes were corrected by melatonin, along with reduction in ER stress and autophagy as well as reversal of sirtuin 1 downregulation [29]. With some likelihood, other data on inflammation may have resulted from transfer of miRNAs. In a study on the immune responsiveness, in vitro differentiated macrophages were exposed to exosomes from hepatocellular carcinoma cells [30]. The cancer cells had either remained untreated or were incubated with melatonin, which obviously changed the composition of the exosomal contents. Exosomes from melatonin-treated cells downregulated in the recipient macrophages the secretion of IL-6, IL-10, IL-1 $\beta$, and TNF- $\alpha$, whereas those from untreated cells upregulated these cytokines [30]. Unfortunately, the responsible miRNAs were not determined.

In the last years, actions of melatonin in progenitor cells have become of increasing interest and some of the respective studies have investigated the role of miRNAs in these effects. The protective, anti-oxidative role of melatonin was recently addressed in cardiac progenitor cells. Premature senescence of these cells was induced by hydrogen peroxide. This was prevented by melatonin, which safeguarded the expression of the IncRNA H19 and its derivative miR-675 [31]. In hair follicle cells of Cashmere goats, melatonin changed the relative expression of several miRNAs, in particular, let-7a, miR-96, miR-199a, miR-205, miR-203, miR-96, miR-199a, miR-96, miR-183, miR-183, and miR-199a [32]. In the murine spermatogonia cell line GC-1spg, melatonin-induced proliferation was suppressed by miR-16, which targets cyclin D1 mRNA [33]. In the same cell line, effects of melatonin on miRNAs were recently studied on the basis of deep sequencing [34]. The consequences were, in fact, profound, as 171 miRNAs were upregulated by melatonin, whereas 5 others were downregulated.

Melatonin is also known to exert oncostatic actions [35-37]. In this context, melatonin was reported to downregulate miR-155 in several human glioma cell lines, an effect that reduced $c-m y b$ (myeloblastosis protooncogene) expression, proliferation and migration [38]. In hypoxic PC-3 prostate cancer cells, melatonin was shown to inhibit angiogenesis, an effect of importance to cancer growth that was concluded to be mediated by miR-3195 and miR-374b [39]. A more detailed study in MCF-7 breast cancer cells revealed alterations in the expression of 22 miRNAs by either $1 \mathrm{nM}$ or $100 \mathrm{nM}$ melatonin [40]. Twelve miRNAs were significantly upregulated and ten others downregulated. According to the analysis of their 5'-utr sequences, the 22 miRNAs should be capable of targeting the remarkable number of 2029 mRNAs [40].

\section{Conclusion}

The study of interaction between melatonin and miRNAs is still in its infancy. To date, the available knowledge does not yet describe a coherent picture, since respective investigations are scattered over the various topics of melatonin research. Nevertheless, the findings already obtained are highly encouraging for continuing research more systematically in this direction. Moreover, it is important to remain aware of the remarkable, previously unforeseen potential of miRNA distribution via exosomes and ectosomes. While these processes may participate in the intraorganismal spreading of pathologies, one might, on the other hand, assume that variations in composition of exosomal and ectosomal miRNAs, if modulated by melatonin, will also spread beneficial or even curative messages within the body.

\section{References}

1. Korkmaz A, Rosales-Corral S, Reiter RJ (2012) Gene regulation by melatonin linked to epigenetic phenomena. Gene 503: 1-11.

2. Hardeland R (2014) Melatonin, noncoding RNAs, messenger RNA stability and epigenetics-evidence, hints, gaps and perspectives. Int J Mol Sci 15: 18221-18252.

3. Hardeland R (2017) Melatonin and the pathologies of weakened or dysregulated circadian oscillators. J Pineal Res 62: e12377.

4. Bartel DP (2009) MicroRNAs: Target recognition and regulatory functions. Cell 136: 215-233.

5. Carthew RW, Sontheimer EJ (2009) Origins and mechanisms of miRNAs and siRNAs. Cell 136: 642-655.

6. Krol J, Loedige I, Filipowicz W (2010) The widespread regulation of microRNA biogenesis, function and decay. Nat Rev Genet 11: 597-610.

7. Da Costa Martins PA, De Windt LJ (2012) Targeting microRNA targets. Circ Res 111: 506-508. 
8. Meunier J, Lemoine F, Soumillon M, Liechti A, Weier M, et al. (2013) Birth and expression evolution of mammalian microRNA genes. Genome Res 23: 34-45.

9. Hardeland R (2017) Intercellular communication via exosomal and ectosomal microRNAs: Facing a jungle of countless microRNAs and targets. In: (Hardeland R (ed) Mini-Reviews in Recent Melatonin Research, Cuvillier, Goettingen, Germany. pp: 109-122.

10. Liu HY, Huang CM, Hung YF, Hsueh YP (2015) The microRNAs Let7c and miR21 are recognized by neuronal Toll-like receptor 7 to restrict dendritic growth of neurons. Exp Neurol 269: 202-212.

11. Paschon V, Takada SH, Ikebara JM, Sousa E, Raeisossadati R, et al. (2016) Interplay between exosomes, microRNAs and toll-like receptors in brain disorders. Mol Neurobiol 53: 2016-2028.

12. Meldolesi J (2016) Ectosomes and exosomes-Two extracellular vesicles that differ only in some details. Biochem Mol Biol J 2: 3.

13. Hansen TB, Jensen TI, Clausen BH, Bramsen JB, Finsen B, et al. (2013) Natural RNA circles function as efficient microRNA sponges. Nature 495: 384-388.

14. Ahadi A, Brennan S, Kennedy PJ, Hutvagner G, Tran N (2016) Long non-coding RNAs harboring miRNA seed regions are enriched in prostate cancer exosomes. Sci Rep 6: 24922.

15. Hardeland R, Cardinali DP, Srinivasan V, Spence DW, Brown GM, et al. (2011) Melatonin-A pleiotropic, orchestrating regulator molecule. Prog Neurobiol 93: 350-384.

16. Hardeland R, Madrid JA, Tan DX, Reiter RJ (2012) Melatonin, the circadian multioscillator system and health: the need for detailed analyses of peripheral melatonin signaling. J Pineal Res 52: 139-166.

17. Ben-Moshe Z, Alon S, Mracek P, Faigenbloom L, Tovin A, et al. (2014) The light-induced transcriptome of the zebrafish pineal gland reveals complex regulation of the circadian clockwork by light. Nucleic Acids Res 42: 3750-3767.

18. Wang X, Tina G, Li Z, Zheng L (2015) The cross-talk between miRNA and mammalian circadian clock. Curr Med Chem 22: 1582-1588.

19. Gao Q, Zhou L, Yang SY, Cao JM (2016) A novel role of microRNA 17-5p in the modulation of circadian rhythm. Sci Rep 6: 30070.

20. Acuña-Castroviejo D, Escames G, Venegas C, Díaz-Casado ME, Lima-Cabello E, et al. (2014) Extrapineal melatonin: sources, regulation and potential functions. Cell Mol Life Sci 71: 2997-3025.

21. Bubenik GA (2002) Gastrointestinal melatonin: Localization, function, and clinical relevance. Dig Dis Sci 47: 2336-2348.

22. Zhu HQ, li Q, Dong LY, Zhou Q, Wang H, et al. (2014) MicroRNA-29b promotes high-fat diet-stimulated endothelial permeability and apoptosis in apoE knock-out mice by downregulating MT1 expression. Int J Cardiol 176: 764-770.

23. Kim YD, Hwang SL, Lee EJ, Kim HM, Chung MJ, et al. (2017) Melatonin ameliorates alcohol-induced bile acid synthesis by enhancing miR-497 expression. J Pineal Res 62: 12386.

24. Kim SJ, Kang HS, Lee JH, Park JH, Jung CH, et al. (2015) Melatonin ameliorates ER stress-mediated hepatic steatosis through miR-23a in the liver. Biochem Biophys Res Commun 458: 462-469.

25. Wu N, Meng F, Zhou T, Han Y, Kennedy L, et al. (2017) Prolonged darkness reduces liver fibrosis in a mouse model of primary sclerosing cholangitis by miR-200b down-regulation. FASEB J 31: 4305-4324.

26. Hardeland R, Cardinali DP, Brown GM, Pandi-Perumal SR (2015) Melatonin and brain inflammaging. Prog Neurobiol 127-128: 46-63.

27. Wang X, Wang ZH, Wu YY, Tang H, Tan L, et al. (2013) Melatonin attenuates scopolamine-induced memory/synaptic disorder by rescuing EPACs/miR-124/Egr1 pathway. Mol Neurobiol 47: 373-381.

28. Zhao Y, Zhao R, Wu J, Wang Q, Pang K, et al. (2018) Melatonin protects against $A \beta$-induced neurotoxicity in primary neurons via miR-132/PTEN/AKT/FOXO3a pathway. Biofactors. doi: 10.1002/biof.1411.

29. Carloni S, Favrais G, Saliba E, Albertini MC, Chalon S, et al. (2016) Melatonin modulates neonatal brain inflammation through endoplasmic reticulum stress, autophagy, and miR-34a/silent information regulator 1 pathway. J Pineal Res 61: 370-380.

30. Cheng L, Liu J, Liu Q, Liu Y, Fan L, et al. (2017) Exosomes from melatonin treated hepatocellularcarcinoma cells alter the immunosuppression status through STAT3 pathway in macrophages. Int J Biol Sci 13: 723-734.

31. Cai B, Ma W, Bi C, Yang F, Zhang L, et al. (2016) Long noncoding RNA H19 mediates melatonin inhibition of premature senescence of c-kit+ cardiac progenitor cells by promoting miR-675. J Pineal Res 61: 82-95.

32. Fu S, Zhao H, Zheng Z, Li J, Zhang W (2014) Melatonin regulating the expression of miRNAs involved in hair follicle cycle of cashmere goats skin. Yi Chuan 36: 1235-1242.

33. Li C, Chen S, Li H, Chen L, Zhao Y, et al. (2016) MicroRNA-16 modulates melatonin-induced cell growth in the mouse-derived spermatogonia cell line GC-1 spg cells by targeting Cond1. Biol Reprod 95: 57.

34. Zhu X, Chen S, Jiang $Y, X u Y$, Zhao $Y$, et al. (2018) Analysis of miRNA expression profiles in melatonin-exposed GC-1 spg cell line. Gene 642: 513-521.

35. Pandi-Perumal SR, Srinivasan V, Maestroni GJ, Cardinali DP, Poeggeler B, et al. (2006) Melatonin: Nature's most versatile biological signal? FEBS J 73: 2813-2838.

36. Blask DE, Dauchy RT, Brainard GC, Hanifin JP (2009) Circadian stage-dependent inhibition of human breast cancer metabolism and growth by the nocturnal melatonin signal: consequences of its disruption by light at night in rats and women. Integr Cancer Ther 8: 347-353.

37. Li Y, Li S, Zhou Y, Meng X, Zhang JJ, et al. (2017) Melatonin for the prevention and treatment of cancer. Oncotarget 8 : 39896-39921.

38. Gu J, Lu Z, Ji C, Chen Y, Liu Y, et al. (2017) Melatonin inhibits proliferation and invasion via repression of miRNA-155 in glioma cells. Biomed Pharmacother 93: 969-975.

39. Sohn EJ, Won G, Lee J, Lee S, Kim SH (2015) Upregulation of miRNA3195 and miRNA374b mediates the anti-angiogenic properties of melatonin in hypoxic PC-3 prostate cancer cells. J Cancer 6: 19-28.

40. Lee SE, Kim SJ, Youn JP, Hwang SY, Park CS, et al. (2011) MicroRNA and gene expression analysis of melatonin-exposed breast cancer cell lines indicating involvement of the anticancer effect. J Pineal Res 5: 345-352. 